\title{
高 Streptomycin interferes with conformational coupling between codon recognition and GTPase activation on the ribosome
}

\author{
Kirill B Gromadski \& Marina V Rodnina
}

\begin{abstract}
Aminoacyl-tRNAs (aa-tRNAs) are selected by the ribosome through a kinetically controlled induced fit mechanism. Cognate codon recognition induces a conformational change in the decoding center and a domain closure of the $30 \mathrm{~S}$ subunit. We studied how these global structural rearrangements are related to tRNA discrimination by using streptomycin to restrict the conformational flexibility of the $30 \mathrm{~S}$ subunit. The antibiotic stabilized aa-tRNA on the ribosome both with a cognate and with a near-cognate codon in the A site. Streptomycin altered the rates of GTP hydrolysis by elongation factor Tu (EF-Tu) on cognate and near-cognate codons, resulting in almost identical rates of GTP hydrolysis and virtually complete loss of selectivity. These results indicate that movements within the $30 \mathrm{~S}$ subunit at the streptomycin-binding site are essential for the coupling between base pair recognition and GTP hydrolysis, thus modulating the fidelity of aa-tRNA selection.
\end{abstract}

Recognition of aa-tRNA on the ribosome is a key reaction of the elongation cycle that determines both the speed and the fidelity of translation. Aa-tRNA is delivered to the A site of the ribosome in a ternary complex with EF-Tu and GTP. The anticodon domain of tRNA binds to the small ribosomal subunit $30 \mathrm{~S}$ according to the mRNA codon in the A site. Correct and incorrect aa-tRNAs are discriminated by a kinetically controlled two-step mechanism that uses induced fit to increase selectivity ${ }^{1}$.

Cognate codon recognition induces a conformational change in the decoding center on the $30 \mathrm{~S}$ ribosomal subunit ${ }^{2}$. These structural alterations stabilize the binding of cognate aa-tRNA to the ribosome and are communicated to the functional centers on the $50 \mathrm{~S}$ ribosomal subunit to accelerate two rearrangement steps, GTPase activation of EFTu and accommodation of aa-tRNA in the peptidyl transferase site $e^{3,4}$. Crystallographic analysis has shown that two critical adenine residues, A1492 and A1493 of helix 44 in 16S rRNA, move from their positions inside the helix toward the minor groove of the codon-anticodon complex and form A-minor interactions with the first two base pairs ${ }^{2}$. The orientation of two other bases, G530 in helix 18 and C1054 in helix 34 of $16 \mathrm{~S}$ rRNA, change such that they form contacts with the second and third positions of the codon-anticodon complex and A1492. These local rearrangements in the decoding region cause the anticodon-binding site to close up ${ }^{5}$. After GTP hydrolysis, EF-Tu changes its conformation from the GTP to the GDP form, which releases the acceptor end of aa-tRNA. EF-Tu-GDP dissociates from the ribosome, whereas the $3^{\prime}$ end of the tRNA moves into the peptidyl transferase center on the 50S subunit, is accommodated there and takes part in peptide bond formation with the $\mathrm{P}$ site-bound peptidyl-
tRNA. The rate of tRNA accommodation in the A site and the dissociation constants of the ternary complex and aa-tRNA from the ribosome also depend on the properties of the codon-anticodon duplex ${ }^{1}$.

Comparison of the crystal structures of the $30 \mathrm{~S}$ subunit in complexes with cognate or near-cognate anticodon stem-loop fragments of tRNA (ASLs) suggests that interaction of the correct codonanticodon complexes with the ribosome leads to a global conformational change of the $30 \mathrm{~S}$ subunit and that this rearrangement is impaired by mismatches in the codon-anticodon duplex ${ }^{5}$. On $70 \mathrm{~S}$ ribosomes, such a conformational change may alter the positions of rRNA elements that connect the two subunits (intersubunit bridges) and/or the orientation of the tRNA, thereby communicating the signal from the $30 \mathrm{~S}$ to the $50 \mathrm{~S}$ subunit. However, the functional importance of $30 \mathrm{~S}$ conformational changes, especially with respect to signaling to $50 \mathrm{~S}$, has not yet been demonstrated.

In this study, we studied whether conformational flexibility of the $30 S$ subunit is essential for signal transmission from the decoding center to the functional centers on the 50S subunit. To interfere with conformational transitions within the $30 \mathrm{~S}$ subunit, we used the antibiotic streptomycin, which induces misreading. Streptomycin connects and immobilizes the shoulder (S12 and G530 loop) and the central part of the subunit (helices 27 and 44$)^{6,7}$. Streptomycin stabilizes aa-tRNA binding in the A site ${ }^{8}$, but not nearly to an extent that would explain the level of misreading induced by the antibiotic ${ }^{9}$. We studied the effect of streptomycin on the key steps in the mechanism of aa-tRNA binding to the A site-that is, ternary complex dissociation versus GTP hydrolysis and aa-tRNA rejection versus accommodation (Fig. 1). The rates of these reactions determine the recognition and 

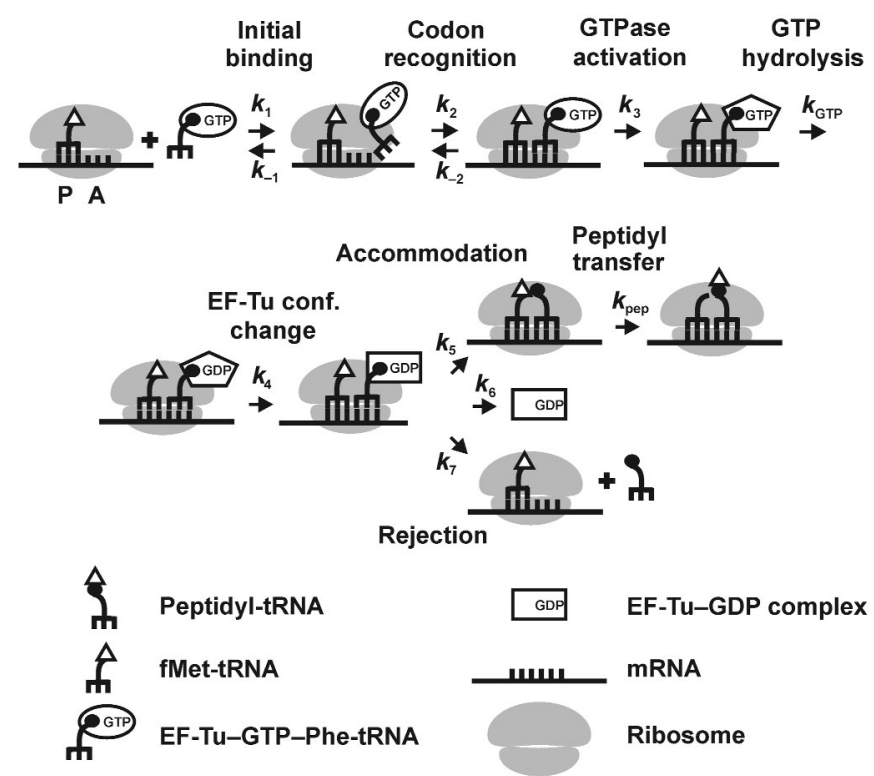

Figure 1 Kinetic scheme of EF-Tu-dependent aa-tRNA binding to the ribosomal A site. Kinetically resolved steps are indicated by numbered rate constants; the chemistry steps that are rate-limited by the preceding step are designated $k_{\mathrm{GTP}}$ and $k_{\mathrm{pep}}$. EF-Tu is shown in different conformations in GTP- and GDP-bound states and in the activated GTPase state.

discrimination of tRNA during the two selection phases, initial selection and proofreading, respectively. The effect of streptomycin on the rate constants was determined using Phe-tRNA ${ }^{\text {Phe }}$ (anticodon $3^{\prime}$-AAG-5') and ribosomes programmed with mRNA composed of an AUG start codon followed by a cognate (UUU) or a near-cognate (CUC) codon in the A site.

\section{RESULTS}

\section{Effect of streptomycin on the key selection steps}

The rate constants that determine the fidelity of tRNA selection are $k_{-2}$ and $k_{3}$ during initial selection and $k_{5}$ and $k_{7}$ during proofreading ${ }^{3}$ (Fig. 1). Rate constants were determined under conditions of high fidelity in a buffer system containing $\mathrm{Mg}^{2+}$ at low concentration $(3.5 \mathrm{mM})$ and the polyamines spermidine $(0.5 \mathrm{mM})$ and putrescine (8 mM) (ref. 10). The overall selectivity (ratio of correct amino acids to incorrect amino acids incorporated) in this system was 450 , compared with 70 in the low-fidelity system used previously ${ }^{3,10}$. This selectivity is well within the range estimated in vivo for Escherichia coli, between 250 and $10^{6}$ (ref. 1). The actual error frequency of the incorporation of phenylalanine for leucine, the amino acids studied in the present work, has not been determined in vivo.

The effect of streptomycin on the association and dissociation rate constants of the initial binding complex, $k_{1}$ and $k_{-1}$, were determined in a model system with fluorescent ternary complex, EF-Tu-GTP-Phe-tRNA ${ }^{\text {Phe }}$ (Prf16/17), and nonprogrammed 70S ribosomes ${ }^{11,12}$. Time courses were measured by fluorescence stopped-flow at increasing ribosome concentrations (up to $1.8 \mu \mathrm{M}$ ), and apparent rate constants were calculated by exponential fitting. From the slope and the $y$-axis intercept of the linear concentration dependence of apparent rate constants (data not shown), rate constants $k_{1}$ and $k_{-1}$ were determined. In the presence of streptomycin, ternary complex binding to the ribosome was slightly faster than in the absence of the antibiotic, whereas the dissociation was not affected (Table 1).
To determine values of the rate constant of codon recognition, $k_{2}$, time courses were measured with EF-Tu-GTP-Phe-tRNA ${ }^{\text {Phe }}$ (Prf16/17) and mRNA-programmed 70S ribosomes with the UUU or CUC codon in the A site and fMet-tRNA ${ }^{\text {fMet }}$ bound to the $\mathrm{P}$ site. Apparent rate constants of the step corresponding to codon recognition were measured at increasing ribosome concentrations (Fig. 2a) and the rate constants calculated as described ${ }^{10}$. From the hyperbolic fits of concentration dependence, the apparent rate constants at ribosome saturation were estimated as $190 \mathrm{~s}^{-1}$ (cognate UUU codon, without streptomycin) ${ }^{10}, 40 \mathrm{~s}^{-1}$ (UUU, with streptomycin), $270 \mathrm{~s}^{-1}$ (near-cognate CUC, without streptomycin) ${ }^{10}$ and $70 \mathrm{~s}^{-1}$ (CUC, with streptomycin). Because the dissociation rate constant, $k_{-2}$, is very small on the cognate UUU codon (see below), the apparent rate constant at saturation gives the value of $k_{2}$ in the cognate situation ${ }^{10}$ (Table 1). In the absence of streptomycin on the CUC codon, $k_{-2}$ is high but $k_{3}$ is negligible, hence the apparent rate constant at saturation gives the sum of $k_{2}+k_{-2}$ (ref. 10). Using the values of $k_{-2}$ determined below, $k_{2}$ was calculated as $190 \pm 30$ $\mathrm{s}^{-1}$ (Table 1). In the presence of streptomycin on the CUC codon, the values of $k_{-2}$ and $k_{3}$ (see below), are small relative to the measured apparent rate constant, $70 \mathrm{~s}^{-1}$, which therefore represents an approximate value of $k_{2}$ (Table 1). Thus, streptomycin reduced the rates of both cognate and near-cognate codon recognition by a factor of three to five.

The rate constant of ternary complex dissociation from the codonrecognition complex, $k_{-2}$, was measured using the GTPase-defective mutant EF-Tu H84A ${ }^{10,13}$. The dissociation of the fluorescent ternary complex, EF-Tu(H84A)-GTP-Phe-tRNA ${ }^{\text {Phe }}$ (Prf16/17), from the codon-recognition complex was initiated by adding nonfluorescent ternary complex in excess (Fig. 2b). In the absence of antibiotic, $k_{-2}$ values were $0.23 \pm 0.05 \mathrm{~s}^{-1}$ (cognate UUU codon) and $80 \pm 10 \mathrm{~s}^{-1}$ (near-cognate CUC), as determined previously ${ }^{10}$. In the presence of streptomycin, cognate and near-cognate complexes were stabilized to a similar extent (Fig. 2b); that is, $k_{-2}$ was decreased 10 -fold (UUU) and 20-fold (CUC) (Table 1).

GTPase activation determines the efficiency of initial selection, limiting the rate of GTP hydrolysis ${ }^{3,14}$. The rate constant of GTPase activation can be determined by measuring GTP hydrolysis rates using $\left[\gamma_{-}{ }^{32} \mathrm{P}\right] \mathrm{GTP}$. In the presence of streptomycin, the rates of GTP hydro-lysis, which differed substantially between cognate and nearcognate ternary complex, were lower on the cognate and higher on the near-cognate codon (Fig. 2c). Values for the rate constant of

Table 1 Effect of streptomycin on the rate constants of ternary complex binding

\begin{tabular}{|c|c|c|c|c|}
\hline \multirow[t]{3}{*}{ Step ${ }^{a}$} & \multicolumn{4}{|c|}{ Rate constant $\left(\mathrm{s}^{-1}\right)^{\mathrm{b}}$} \\
\hline & \multicolumn{2}{|c|}{ UUU } & \multicolumn{2}{|c|}{ CUC } \\
\hline & - Str & + Str & - Str & + Str \\
\hline \multicolumn{5}{|l|}{ Initial binding } \\
\hline$k_{1}\left(\mu \mathrm{M}^{-1} \mathrm{~s}^{-1}\right)$ & $140 \pm 20$ & $190 \pm 20$ & $140 \pm 20$ & $190 \pm 20$ \\
\hline$k_{-1}\left(\mu \mathrm{M}^{-1} \mathrm{~s}^{-1}\right)$ & $85 \pm 25$ & $80 \pm 25$ & $85 \pm 25$ & $80 \pm 25$ \\
\hline \multicolumn{5}{|c|}{ Codon recognition } \\
\hline$k_{2}\left(\mu \mathrm{M}^{-1} \mathrm{~s}^{-1}\right)$ & $190 \pm 20$ & $40 \pm 10$ & $190 \pm 30$ & $70 \pm 20$ \\
\hline$k_{-2}\left(\mu \mathrm{M}^{-1} \mathrm{~s}^{-1}\right)$ & $0.23 \pm 0.05$ & $\leq 0.01$ & $80 \pm 10$ & $5.0 \pm 0.5$ \\
\hline \multicolumn{5}{|c|}{ GTPase activation and GTP hydrolysis ${ }^{c}$} \\
\hline$k_{3}\left(\mu \mathrm{M}^{-1} \mathrm{~s}^{-1}\right)$ & $250 \pm 60$ & $2.2 \pm 0.5$ & $0.4 \pm 0.1$ & $2.1 \pm 0.6$ \\
\hline
\end{tabular}

Effect of streptomycin on ternary complex binding to cognate (UUU) or near-cognate (CUC) codons in the A site. Str, streptomycin.

aSteps are defined in Figure 2. ${ }^{b}$ Rate constants of reactions in the absence of streptomycin are taken from ref. 10. 'Rearrangement limits the rate of the chemical step. 
Figure 2 Effect of streptomycin on the kinetics of A-site binding. (a) Determination of $k_{2}$. Ternary complex EF-Tu-GTP-Phe-tRNA Phe (Prf16/17) (anticodon $3^{\prime}$-AAG-5') (0.1 $\mu \mathrm{M}$ after mixing) was added in the presence of streptomycin $(20 \mu \mathrm{M})$ to mRNA-programmed ribosomes with fMet-tRNA ${ }^{\text {fMet }}$ in the P site and the cognate UUU or near-cognate CUC codon in the A site. (b) Determination of $k_{-2}$. The codon-recognition complex was prepared by mixing EF-Tu(H84A)GTP-Phe-tRNAPhe(Prf16/17) and mRNAprogrammed ribosomes with $\mathrm{fMet}$-tRNA $\mathrm{fMet}^{\mathrm{fM}}$ in the $\mathrm{P}$ site $(0.3 \mu \mathrm{M}$ each after mixing). Dissociation of the complex was initiated by adding a ten-fold excess of nonfluorescent EF-Tu(H84A)-GTPPhe-tRNA Phe. Time courses were measured by stopped-flow on the cognate UUU codon in the absence (1) and presence (2) of streptomycin $(20 \mu \mathrm{M})$, or on the near-cognate CUC codon in the absence (3) and presence (4) of antibiotic; no dissociation was observed upon addition of buffer alone (5). (c) GTP hydrolysis. Time courses of $\left[\gamma^{32}\right.$ P] GTP hydrolysis in the ternary complex $(0.2 \mu \mathrm{M})$ on mRNA-programmed ribosomes $(2.8 \mu \mathrm{M})$. ( $\mathbf{\square})$, no ribosomes. (d) Determination of $k_{3}$. Concentration dependence of the apparent rate constants of GTP hydrolysis in ribosomebound ternary complex, $k_{\mathrm{app}}$. (e) Peptide bond formation. Extent of fMetPhe formation on UUU with or without streptomycin or CUC with streptomycin is shown on the left ordinate, that on CUC without antibiotic $(\boldsymbol{\Lambda})$ on the right ordinate. Time courses of $\mathrm{f}\left[{ }^{3} \mathrm{H}\right]$ Met $\left[{ }^{14} \mathrm{C}\right]$ Phe formation were measured by quench-flow upon mixing ternary complex $(0.2 \mu \mathrm{M})$ with ribosomes $(1 \mu \mathrm{M})$.
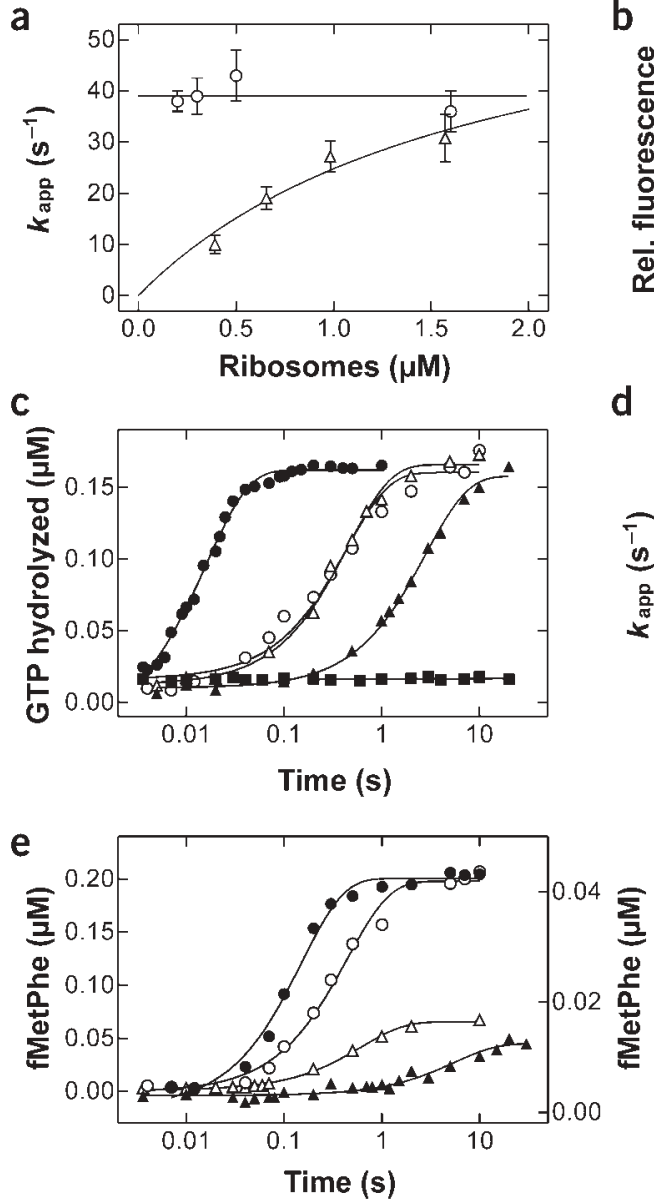

b

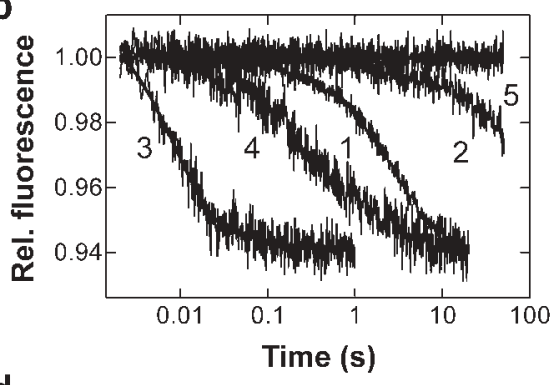

d

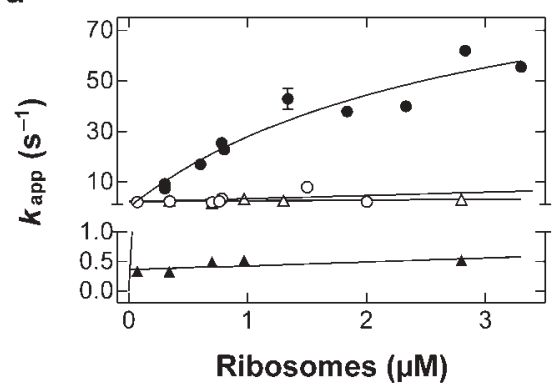

GTPase activation, $k_{3}$, were determined from the concentration dependence of the respective apparent rate constants ${ }^{10}$ (see Methods; Fig. 2d). In the presence of streptomycin, the rate constants of GTPase activation became equal on cognate and nearcognate codons (Table 1). The inhibition by streptomycin of GTP hydrolysis in cognate ternary complex has been noted previously, but rate constants were not measured ${ }^{15}$. Our results suggest that streptomycin interferes with conformational changes within or near the decoding center that are induced by cognate codon recognition and locks the ribosome in a conformation that has slow communication between subunits (slow GTPase) and is un-selective, because the rate of GTPase activation is no longer different on cognate and near-cognate codons.

The streptomycin effect on peptide bond formation was similar (Fig. 2e). Apparent rate constants of the reaction were $6 \mathrm{~s}^{-1}$ (UUU, no antibiotic), $2.1 \mathrm{~s}^{-1}$ (UUU, with streptomycin), $0.3 \mathrm{~s}^{-1}$ (CUC, no antibiotic) and $1.7 \mathrm{~s}^{-1}$ (CUC, with streptomycin). In the presence of streptomycin, rates of peptide bond formation were lower on the cognate codon and higher on the near-cognate codon. Rate constants of aatRNA accommodation and rejection ( $k_{5}$ and $k_{7}$, respectively) could not be determined, because in the presence of streptomycin the slow GTPase activation was rate limiting for those steps.

\section{Interplay between streptomycin and paromomycin}

The kinetic results indicate that the mode of action of streptomycin is different from that of other antibiotics that induce misreading, notably paromomycin, which switches the ribosome into a high- affinity and high-activity conformation regardless of whether cognate or near-cognate aa-tRNA is bound to the A site ${ }^{2,4}$. Kinetic measurements indicate that paromomycin accelerates the GTPase activation step in the near-cognate ternary complex up to the cognate value ${ }^{4}$, whereas GTP hydrolysis in the cognate complex remains high and rate-limited by the preceding codon recognition step (T. Pape, Imperial College London, UK, and M.V.R., unpublished data). Paromomycin induces a local conformational change in the decoding region that is similar to, and enhanced by, the change induced by cognate codon recognition ${ }^{2}$, and a global rearrangement of the $30 \mathrm{~S}$ subunit that causes the decoding site to close ${ }^{5}$. Thus, we next tested whether the combined effect of cognate codon recognition and paromomycin binding override the effect of streptomycin. First, we verified by chemical footprinting that streptomycin and paromomycin bind simultaneously to the ribosome (Fig. 3a), as expected from crystal structures ${ }^{2,6}$ and previous antibiotic-binding studies ${ }^{16}$. Then, we compared the rates of GTP hydrolysis on cognate and near-cognate codons in the presence of either paromomycin or streptomycin, or with both antibiotics together (Fig. 3b). As expected ${ }^{4}$, in the presence of paromomycin alone, the rate of GTP hydrolysis was not affected on the cognate and was increased on the near-cognate codon, although not to the same extent as in the experimental system used earlier ${ }^{4}$. In the presence of streptomycin alone, GTP hydrolysis was slow and independent on the codon-anticodon mismatch, as shown above. In the presence of both antibiotics, the rate of GTP hydrolysis in the cognate ternary complex remained as low as in the presence of streptomycin alone. These data indicate that streptomycin locks the ribosome into a 
a

b
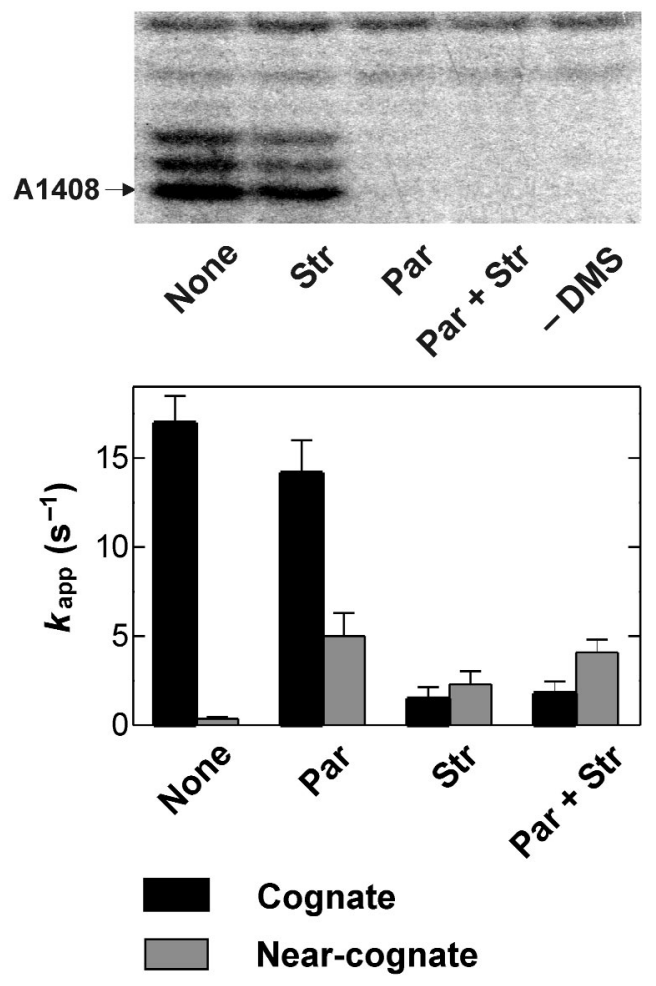

structure that is unable to respond in a normal way to both paromomycin and binding of the cognate tRNA.

On the near-cognate codon, the rates of GTP hydrolysis induced by streptomycin and paromomycin alone or together were similar at $2-5 \mathrm{~s}^{-1}$. Also in this case, streptomycin seemed to reduce the rate of GTP hydrolysis induced by paromomycin; however, these experiments were not precise enough to distinguish the effects of the two antibiotics.

\section{Streptomycin impairs both selection steps}

Reports of streptomycin's effect on the fidelity of aa-tRNA selection are contradictory: streptomycin has been reported to influence initial selection only ${ }^{17}$, proofreading only ${ }^{9,15,18-20}$, or both ${ }^{14}$. To clarify which selection step is affected, we determined the efficiencies of initial selection and proofreading separately in the presence and absence of streptomycin, using ribosomes with CUC coding for leucine in the A site. As the overall selectivity of amino acid incorporation must be measured in the presence of competing substrates ${ }^{21}$, cognate and nearcognate ternary complexes were added in excess over ribosomes. The efficiency of proofreading was calculated from the distribution of aa-tRNA among accommodation and peptide bond formation and rejection reactions in the absence of a competitor ${ }^{3,22,23}$. Finally, the efficiency of initial selection was calculated from the ratio of overall selectivity and proofreading ${ }^{21}$.

Overall selectivity was measured in the presence of the ternary complexes containing CUC-specific Leu-tRNA ${ }^{\text {Leu }}$ as cognate and Phe-

Figure 4 Discrimination of aa-tRNA. Selectivity was measured in the absence of antibiotic (black bars) or in the presence of $20 \mu \mathrm{M}$ streptomycin (gray bars). Overall selectivity was measured on CUC-programmed ribosomes in the presence of excess ternary complexes containing equal amounts of Leu- and Phe-tRNA. Proofreading was measured with EF-Tu-GTP-PhetRNA ${ }^{\text {Phe }}$ alone in excess of CUC-programmed ribosomes. Initial selection was calculated from the overall selectivity and proofreading as described in Methods.
Figure 3 Effect of streptomycin and paromomycin on GTP hydrolysis. (a) Chemical probing experiment showing protection of A1408 in 16S rRNA by paromomycin $(50 \mu \mathrm{M})$ from modification with dimethyl sulfate ${ }^{46}$ in the absence or presence of streptomycin $(20 \mu \mathrm{M})$. None, no antibiotics added; -DMS, control without dimethyl sulfate modification. (b) Apparent rate constants of GTP hydrolysis were measured with ternary complex $(0.2 \mu \mathrm{M})$ and ribosomes $(0.8 \mu \mathrm{M})$ programmed by the cognate UUU (black bars) or near-cognate CUC (gray bars) codons without antibiotic or in the presence of paromomycin $(50 \mu \mathrm{M})$, or streptomycin $(20 \mu \mathrm{M})$, or both.

tRNA $^{\text {Phe }}$ as near-cognate substrates. Ternary complexes were added in excess over ribosomes, and the formation of correct and incorrect dipeptides, fMetLeu and fMetPhe, respectively, was measured. The overall selectivity in the absence of streptomycin was 450 (only one in 450 dipeptides was incorrect; Fig. 4). Streptomycin abolished the selectivity of the ribosome nearly completely, resulting in the incorporation of 1 incorrect for every 3.7 correct amino acids. Thus, streptomycin reduced the overall selectivity $>100$-fold.

To measure the efficiency of proofreading, excess ribosomes with a CUC codon in the A site were mixed with EF-Tu-GTP-Phe-tRNA ${ }^{\text {Phe }}$ (near-cognate), and the formation of fMetPhe dipeptide was monitored. Under these conditions - in the absence of cognate competitorthere is no initial selection because all near-cognate ternary complexes present can enter the ribosome and eventually hydrolyze GTP, and the only source of selectivity is rejection of near-cognate Phe-tRNA ${ }^{\text {Phe }}$ during the proofreading phase. Analogous experiments using the cognate UUU codon were done in parallel. From the extent of phenylalanine incorporation into dipeptides on cognate and near-cognate codons, the selectivity of proofreading can be calculated ${ }^{21}$. According to these data, streptomycin decreased the selectivity of proofreading about ten-fold, from 15 to 1.4 (Fig. 4). We determined the efficiency of proofreading from the ratio of GTP hydrolyzed to dipeptide formed ${ }^{22}$ and obtained the same results (data not shown). From the values of overall selectivity and proofreading determined experimentally, the efficiency of initial selection can be calculated, because overall selectivity is given by the multiplied selectivities of initial selection and proofreading ${ }^{21}$. According to this calculation, initial selection was diminished ten-fold, from 27 to 2.6 (Fig. 4). Thus, streptomycin impaired both selection steps, initial selection and proofreading, to the same extent.

\section{DISCUSSION}

The present kinetic analysis shows that the rate of GTPase activation of ribosome-bound ternary complex is strongly affected when the

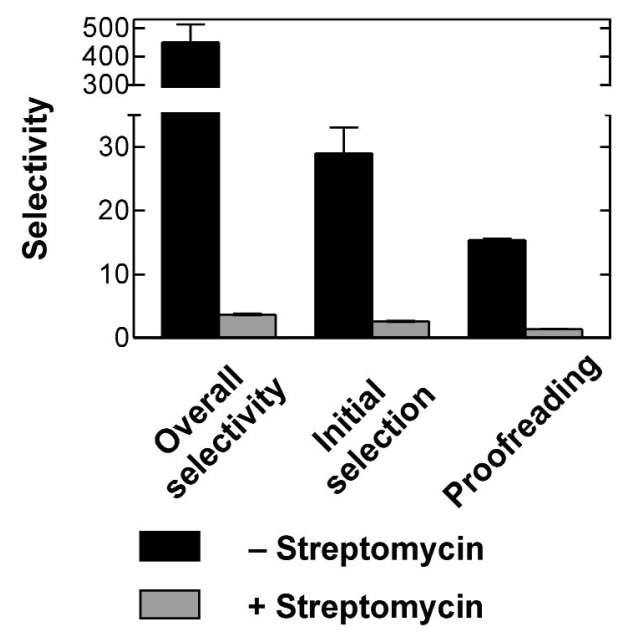


conformational flexibility of the $30 \mathrm{~S}$ subunit is restricted by streptomycin binding. This indicates that conformational transitions of the 30 S subunit at or near the streptomycin-binding site are important in the regulation of the rate of GTP hydrolysis by EF-Tu, which, in turn, determines the accuracy of aa-tRNA selection ${ }^{10}$. In the presence of streptomycin the rates of GTP hydrolysis are no longer different for cognate and near cognate ternary complexes hence the loss of selectivity caused by the antibiotic. This effect suggests that streptomycin stabilizes the ribosome in a conformation that sustains moderate GTPase activation independent of whether there is cognate or near-cognate codon recognition, but is insufficient to achieve the rapid GTPase activation induced by cognate codon recognition in the absence of antibiotic. The effect of streptomycin cannot be reversed by paromomycin binding in the decoding site, which otherwise strongly enhances GTPase activation, in situations where (nearcognate) codon recognition is too weak to induce full GTPase activation. Thus, the present kinetic data strongly indicate that movements of parts of the 30 subunit $^{5}$ are essential for the specific and rapid conformational coupling between the subunits that regulates GTP hydrolysis by EF-Tu.

Crystal structures suggest that local conformational changes at the decoding site, as induced by cognate codon recognition, lead to a global change in the structure of the $30 \mathrm{~S}$ subunit from an open to a closed form ${ }^{7}$. The change involves a rotation of the $30 \mathrm{~S}$ shoulder toward the intersubunit space and the region comprising helices 44 and 27 and the platform ${ }^{5}$. The present kinetic data suggest that streptomycin locks the ribosome in a conformation different from that induced by cognate tRNA or paromomycin. In fact, according to the crystal structures, streptomycin connects the shoulder to the central part of the subunit and induces closing of the $30 \mathrm{~S}$ subunit, albeit not into precisely the same position as that resulting from cognate codon-anticodon recognition ${ }^{6}$. Streptomycin seems to stabilize a particular 30 S conformation, thereby precluding the specific structural change that correlates with the high GTPase rate characteristic of cognate codon recognition. This suggests that the structural change communicates the local conformational change in the decoding site, as induced by cognate codon recognition, to the GTPase-activating centers on the $50 \mathrm{~S}$ subunit. This contention is supported by genetic data suggesting that mutations in the shoulder region affect translational fidelity ${ }^{24-27}$. Closing of the $30 \mathrm{~S}$ head may also have an effect ${ }^{28}$, although most of the head movements seem to take place upon binding of the $50 \mathrm{~S}$ subunit ${ }^{29}$. The present data support the suggestion that $30 \mathrm{~S}$ domain closure provides the structural link among codon recognition, GTP hydrolysis and aa-tRNA accommodation, thus modulating the fidelity of tRNA selection ${ }^{7}$.

Near-cognate anticodon stem-loop tRNA fragments alone, without paromomycin, cannot induce rapid GTP hydrolysis ${ }^{3,10}$. The closed conformation of the 30 S subunit does not seem to form; as in crystals of the respective $30 \mathrm{~S}$ complexes, the shoulder does not move and the head moves in a different direction ${ }^{5}$. Binding of paromomycin accelerates GTP hydrolysis by EF-Tu when a near-cognate substrate is bound $^{4}$, enhances local rearrangements at the decoding center ${ }^{2}$ and induces the formation of the closed form of the $30 \mathrm{~S}$ subunit that otherwise requires cognate codon recognition ${ }^{5}$. The conformational change induced by paromomycin probably depends on the conditions and type of mismatch. With a $\mathrm{U} \bullet \mathrm{G}$ mismatch at the first codon position, the transition to the closed form is almost complete, as is suggested by the crystal structures ${ }^{5}$. Under the present conditions $(\mathrm{C} \bullet \mathrm{A}$ mismatch at the first codon position and low $\mathrm{Mg}^{2+}$ concentration), the global transition seems to take place only partially, because in the presence of paromomycin the GTPase rate in the near-cognate ternary complex is slower than that in the cognate. A streptomycin-induced conformational change of the $30 \mathrm{~S}$ also leads to acceleration of GTP hydrolysis in the near-cognate ternary complex. This also suggests that particular movements of the $30 \mathrm{~S}$ subunit are essential for the communication between the decoding center of the ribosome and the GTPase center in EF-Tu, and that different misreading-inducing antibiotics act by inducing and stabilizing ribosome conformations that are insensitive to the correct geometry of the codon-anticodon complex.

The mechanism of communication between the decoding and GTPase centers is not known. The $30 \mathrm{~S}$ subunit closure is expected to change the positions of aa-tRNA and of several intersubunit bridges, some of which are located near the EF-Tu-binding site and the peptidyl transferase center on the $50 \mathrm{~S}$ subunit. This suggests that the closure of the 30 S subunit may be transmitted to the 50 S subunit through the intersubunit bridges, or the tRNA molecule in the ternary complex, or both, and lead to the conformational rearrangement in EF-Tu required for GTPase activation ${ }^{1}$. Domain closure of the $30 \mathrm{~S}$ subunit probably influences the contacts of EF-Tu via interactions of S12 with aa-tRNA ${ }^{30-32}$. Direct contacts between helix 5 of $16 \mathrm{~S}$ rRNA and domain II of EF-Tu may also be involved ${ }^{7,32,33}$. In addition to binding of the codon-anticodon complex in the decoding center and the interaction with S12, some interactions between the ternary complex and the ribosome involve contacts of the elbow region of tRNA with the L11 region of the ribosome $e^{30-32}$. The structure of aa-tRNA in the codon-recognition complex is distorted ${ }^{30-32}$, and structural, genetic and kinetic data strongly indicate that this distortion influences tRNA discrimination, most likely by influencing communication between the subunits ${ }^{1,30,31,34-37}$. Freezing the flexibility of the 30 S subunit in the vicinity of the decoding region may inhibit the tRNA rearrangements or change the contacts with the $50 \mathrm{~S}$ subunit, thus reducing the rate of GTP hydrolysis by EF-Tu.

The mechanism by which EF-Tu on the ribosome is activated for GTP hydrolysis is not fully understood. Rapid GTP hydrolysis requires that a residue crucial for catalysis, His 84 in EF-Tu, change its orientation toward the nucleotide-binding pocket where the hydrolytic water molecule is located ${ }^{13,38}$. The respective conformational change of the $\mathrm{G}$ domain seems to be induced by interactions with the ribosome in a concerted manner. First, the interaction with the ribosomal protein $\mathrm{L} 7 / 12$ is important, because the removal of L7/12 from the ribosome reduces the rate of GTP hydrolysis 2,000-fold ${ }^{39}$. Second, the contact of the sarcin-ricin loop of $23 \mathrm{~S}$ rRNA with the switch I and II regions at the nucleotide-binding pocket of EF-Tu may be essential, by analogy to other GTP-binding proteins in which GTPase activation involves a contact between the switch regions and the respective GTPaseactivating protein ${ }^{40}$. Third, the intact aa-tRNA molecule is required to activate GTP hydrolysis ${ }^{37}$, indicating that the interactions of the acceptor arm of aa-tRNA with the switch regions of the factor may be involved. All of these interactions may be affected by conformational changes of the $30 \mathrm{~S}$ subunit that are initiated in the decoding site and transmitted to the G domain of EF-Tu through the tRNA and rRNA bridges connecting the subunits.

\section{METHODS}

Buffer and reagents. Experiments were done in buffer A $(50 \mathrm{mM}$ Tris- $\mathrm{HCl}$, $\mathrm{pH}$ 7.5, $70 \mathrm{mM} \mathrm{NH} \mathrm{Cl}_{4}, 30 \mathrm{mM} \mathrm{KCl}, 3.5 \mathrm{mM} \mathrm{MgCl}_{2}, 0.5 \mathrm{mM}$ spermidine, $8 \mathrm{mM}$ putrescine and $2 \mathrm{mM} \mathrm{DTT}$ ) at $20^{\circ} \mathrm{C}$. Chemicals were obtained from Roche Molecular Biochemicals or Merck. Mant-GTP was purchased from JenaBioScience. Radioactive compounds were obtained from ICN.

Ribosomes, EF-Tu and tRNAs. Ribosomes from E. coli MRE600, wild-type EF-Tu and tRNAs were prepared as described ${ }^{12,36}$. EF-Tu H84A ${ }^{13}$ was expressed and purified using an established protocol ${ }^{41}$. For the kinetic experiments, ternary complex EF-Tu-GTP- $\left[{ }^{14} \mathrm{C}\right] \mathrm{Phe}-\mathrm{tRNA}{ }^{\text {Phe }}$ was prepared by incubating 
$30 \mu \mathrm{M}$ wild-type or H84A EF-Tu, $0.02 \mu \mathrm{M}$ EF-Ts, $1 \mathrm{mM}$ GTP, $3 \mathrm{mM}$ phosphoenol pyruvate, $0.1 \mathrm{mg} \mathrm{ml}^{-1}$ pyruvate kinase, $10 \mu \mathrm{M}$ tRNA ${ }^{\mathrm{Phe}}$ (Prf16/17), $3 \mathrm{mM}$ ATP, $30 \mu \mathrm{M}\left[{ }^{14} \mathrm{C}\right]$ phenylalanine and S100 fraction as a source of Phe-tRNA synthetase $(2 \%(\mathrm{v} / \mathrm{v}))$, and purified by gel filtration as described ${ }^{12,36}$. Ternary complexes containing mant-GTP or $\left[\gamma^{-32} \mathrm{P}\right]$ GTP were prepared in the same way, except that purified $\left[{ }^{14} \mathrm{C}\right] \mathrm{Phe}-\mathrm{tRNA}{ }^{\text {Phe }}(10 \mu \mathrm{M})$ was used and the concentration of nucleotides was $300 \mu \mathrm{M}$ mant-GTP or $30 \mu \mathrm{M}\left[\gamma_{-}{ }^{32} \mathrm{P}\right] \mathrm{GTP}$.

$70 \mathrm{~S}$ initiation complex was prepared in buffer B ( $50 \mathrm{mM}$ Tris- $\mathrm{HCl}, \mathrm{pH} 7.5$, $70 \mathrm{mM} \mathrm{NH}_{4} \mathrm{Cl}, 30 \mathrm{mM} \mathrm{KCl}, 7 \mathrm{mM} \mathrm{MgCl}_{2}, 2 \mathrm{mM} \mathrm{DTT}$ ) by incubating $1 \mu \mathrm{M}$ ribosomes with $\mathrm{f}\left[{ }^{3} \mathrm{H}\right] \mathrm{Met}-\mathrm{tRNA}{ }^{\mathrm{fMet}}$ and $4 \mu \mathrm{M}$ mRNA in the presence of initiation factors IF1, IF2 and IF3 ( $1.5 \mu \mathrm{M}$ each) for $70 \mathrm{~min}$ at $37^{\circ} \mathrm{C}$. mRNAs were derivatives of a 122 -nucleotide $\mathrm{mRNA}^{42,43}$ that included a Shine-Dalgarno sequence, an AUG start codon, and UUU or CUC as the second codon. Ribosome complexes were purified and concentrated by centrifugation through 1.1 M sucrose cushions at 259,000 $\mathrm{g}$ for $2 \mathrm{~h}$ on a Sorvall M120GX ultracentrifuge. The occupancy of both A and P sites was $80-90 \%$, as determined by nitrocellulose filtration.

Kinetic experiments. Fluorescence stopped-flow experiments were done on a SX-18MV spectrometer (Applied Photophysics) as described ${ }^{3,12}$. Proflavin was excited at $470 \mathrm{~nm}$ and its fluorescence measured after passing a KV500 filter (Schott). Experiments were done in buffer $\mathrm{A}$ at $20^{\circ} \mathrm{C}$ by mixing equal volumes of reactants $(60 \mu \mathrm{l}$ each) and monitoring the time courses of fluorescence changes. Time courses shown in the figures were obtained by averaging 3-7 individual transients.

Quench-flow assays were done in a KinTek apparatus. Equal volumes $(14 \mu \mathrm{l})$ of ribosomal complexes and ternary complex were rapidly mixed at $20^{\circ} \mathrm{C}$. To determine the rates of peptide bond formation, reactions were quenched with $0.8 \mathrm{M} \mathrm{KOH}$ and $\mathrm{f}\left[{ }^{3} \mathrm{H}\right] \mathrm{Met}\left[{ }^{14} \mathrm{C}\right]$ Phe dipeptides were analyzed as described ${ }^{44}$. To measure $\left[\gamma_{-}{ }^{32} \mathrm{P}\right]$ GTP hydrolysis, reactions were quenched with $40 \%(\mathrm{v} / \mathrm{v})$ formic acid and analyzed by thin-layer chromatography in $0.5 \mathrm{M}$ potassium phosphate, $\mathrm{pH} 3.5$, using Polygram CEL300 TLC plates.

Apparent rate constants were determined by exponential fitting, using up to three exponential terms (characterized by variable time constant, $k_{\text {app}}$, and respective amplitudes) and a variable for the final signal. Calculations were done and standard deviations for apparent constants $k_{\text {app }}$ were calculated using TableCurve (Jandel Scientific) as described ${ }^{3,10,12} \cdot k_{-2}$ values were determined as shown in Figure $2 \mathbf{b}$. Elemental rate constants $k_{1}, k_{-1}, k_{2}$ and $k_{3}$ were calculated from the concentration dependence of apparent rate constants of initial binding, codon recognition and GTP hydrolysis as described ${ }^{10,13}$. In the absence of streptomycin on UUU, $k_{5}$ and $k_{7}$ were calculated from the apparent rate constant of dipeptide formation, which gives the value for $k_{5}+k_{7}$, and the efficiency of phenylalanine incorporation into fMetPhe dipeptide, which gives $k_{5} /$ $\left(k_{5}+k_{7}\right)$ (ref. 21). In all other cases this calculation was not possible, because the rate of peptide bond formation was limited by the preceding step of GTP hydrolysis. Values for $k_{4}$ and $k_{6}$ were not determined, because the respective reactions do not contribute to aa-tRNA discrimination ${ }^{3}$.

Selectivity measurements. 70S initiation complexes were prepared as described above, except that nonradioactive fMet-tRNA ${ }^{\mathrm{fMet}}$ was used. $\left[{ }^{3} \mathrm{H}\right] \mathrm{Phe}-\mathrm{tRNA}{ }^{\mathrm{Phe}}$ or $\left[{ }^{14} \mathrm{C}\right]$ Leu-tRNA ${ }^{\mathrm{Leu}}$ were prepared using partially purified Phe- or Leu-tRNA synthetases from E. coli and purified by HPLC on a LiChrospher WP300 RP-18 column (Merck). Ternary complexes were prepared by incubating $20 \mu \mathrm{M}$ EF$\mathrm{Tu}, 1 \mathrm{mM}$ GTP, $3 \mathrm{mM}$ phosphoenol pyruvate, $0.1 \mathrm{mg} \mathrm{ml}^{-1}$ pyruvate kinase and $10 \mu \mathrm{M}$ aa-tRNA. To measure the overall selectivity, $70 \mathrm{~S}$ initiation complexes with the CUC codon in the A site $(0.5 \mu \mathrm{M}, 50$ pmol per assay) were mixed with unpurified ternary complexes containing Leu-tRNA specific for the CUC codon $(1.5 \mu \mathrm{M})$ and Phe-tRNA as the near-cognate aa-tRNA $(1.5,2.5$ or $5 \mu \mathrm{M})$. After 1 min incubation at $20^{\circ} \mathrm{C}$, reactions were stopped by adding $0.5 \mathrm{M} \mathrm{KOH}$, incubated $15 \mathrm{~min}$ at $37^{\circ} \mathrm{C}$, neutralized with acetic acid, and dipeptides were analyzed by HLPC on a LiChrospher 100 RP-8 column (Merck). A gradient of $0-65 \%(\mathrm{v} / \mathrm{v})$ acetonitril in $0.1 \%(\mathrm{v} / \mathrm{v})$ TFA was adapted in such a way to separate the peaks of $\left[{ }^{3} \mathrm{H}\right] \mathrm{Phe},\left[{ }^{14} \mathrm{C}\right] \mathrm{Leu}, \mathrm{fMet}\left[{ }^{3} \mathrm{H}\right] \mathrm{Phe}$ and $\mathrm{fMet}\left[{ }^{14} \mathrm{C}\right]$ Leu. Fractions after HPLC were collected and the radioactivity was counted after addition of Lumasafe Plus scintillation cocktail (Packard).

The overall selectivity, $S$, was calculated from the ratio of correct (fMetLeu) to incorrect (fMetPhe) dipeptide formed, normalized by the initial concentra- tions of cognate and near-cognate ternary complexes, respectively: $S=([\mathrm{fMetLeu}] /[$ LeuTC $]) /([\mathrm{fMetPhe}] /[\mathrm{PheTC}])$. Alternatively, selectivity was determined from the ratio of velocities of dipeptide formation with correct and incorrect substrates, $S=V_{\text {Leu }} / V_{\text {Phe }}=\ln (1-[\mathrm{fMetLeu}] /[$ LeuTC $]) / \ln (1-$ [fMetPhe] / [PheTC]). Both methods gave the same results within the experimental error. Proofreading was measured with the same materials, except that only one type of ternary complex, either cognate or near-cognate, was added $(0.25 \mu \mathrm{M})$, and the ribosomes $(0.5 \mu \mathrm{M})$ were in excess. The selectivity of proofreading of near-cognate tRNA, $P$, was calculated from the ratio of dipeptide formed on cognate versus near-cognate codon, corrected for the total amount of ternary complex added, $P=\left(\left[\right.\right.$ fMetPhe $\left._{\mathrm{UUU}}\right] /\left[\right.$ Phe $\left.\left.\operatorname{added}_{\mathrm{UUU}}\right]\right) /$ ([fMetPhe $\left.{ }_{\mathrm{CUC}}\right] /$ [ Phe added $\left._{\mathrm{CUC}}\right]$ ) as described ${ }^{21}$. The efficiency of initial selection, $I$, was calculated from the efficiencies of overall selectivity and proofreading, as determined experimentally, using the equation ${ }^{21} I=S / P$.

Chemical probing. DMS modification of ribosome complexes prepared as described above $(0.3 \mu \mathrm{M})$ was carried out for $10 \mathrm{~min}$ at $37^{\circ} \mathrm{C}$ (ref. 45). Ribosomal RNA was purified by repeated phenol extractions, and methylated sites were determined by primer extension sequencing with AMV reverse transcriptase ${ }^{45}$, using an oligodeoxyribonucleotide primer complementary to positions $1457-1473$ of $16 \mathrm{~S}$ rRNA.

\section{ACKNOWLEDGMENTS}

We thank W. Wintermeyer for critical reading and discussions and V. Ramakrishnan for valuable comments on the manuscript. We thank A. Kubarenko for doing the footprinting experiment, V. Katunin and Y. Semenkov for tRNA preparations, U. Kothe for donating EF-Tu H84A, P. Striebeck, A. Böhm, C. Schillings and S. Möbitz for expert technical assistance. The work was supported by the Deutsche Forschungsgemeinschaft, the Alfried Krupp von Bohlen und Halbach-Stiftung and the Fonds der Chemischen Industrie.

\section{COMPETING INTERESTS STATEMENT}

The authors declare that they have no competing financial interests.

Received 14 December 2003; accepted 10 February 2004

Published online at http://www.nature.com/natstructmolbiol/

1. Rodnina, M.V. \& Wintermeyer, W. Fidelity of aminoacyl-tRNA selection on the ribosome: kinetic and structural mechanisms. Annu. Rev. Biochem. 70, 415-435 (2001).

2. Ogle, J.M. et al. Recognition of cognate transfer RNA by the $30 \mathrm{~S}$ ribosomal subunit. Science 292, 897-902 (2001).

3. Pape, T., Wintermeyer, W. \& Rodnina, M.V. Induced fit in initial selection and proofreading of aminoacyl-tRNA on the ribosome. EMBO J. 18, 3800-3807 (1999).

4. Pape, T., Wintermeyer, W. \& Rodnina, M.V. Conformational switch in the decoding region of $16 \mathrm{~S}$ rRNA during aminoacyl-tRNA selection on the ribosome. Nat. Struct. Biol. 7, 104-107 (2000).

5. Ogle, J.M., Murphy, F.V., Tarry, M.J. \& Ramakrishnan, V. Selection of tRNA by the ribosome requires a transition from an open to a closed form. Cell 111, 721-732 (2002).

6. Carter, A.P. et al. Functional insights from the structure of the $30 \mathrm{~S}$ ribosomal subunit and its interactions with antibiotics. Nature 407, 340-348 (2000).

7. Ogle, J.M., Carter, A.P. \& Ramakrishnan, V. Insights into the decoding mechanism from recent ribosome structures. Trends Biochem. Sci. 28, 259-266 (2003).

8. Karimi, R. \& Ehrenberg, M. Dissociation rate of cognate peptidyl-tRNA from the Asite of hyper- accurate and error-prone ribosomes. Eur. J. Biochem. 226, 355-360 (1994).

9. Ruusala, T. \& Kurland, C.G. Streptomycin preferentially perturbs ribosomal proofreading. Mol. Gen. Genet. 198, 100-104 (1984).

10. Gromadski, K.B. \& Rodnina, M.V. Kinetic determinants of high-fidelity tRNA discrimination on the ribosome. Mol. Cell 13, 191-200 (2004).

11. Rodnina, M.V., Pape, T., Fricke, R., Kuhn, L. \& Wintermeyer, W. Initial binding of the elongation factor Tu-GTP-aminoacyl-tRNA complex preceding codon recognition on the ribosome. J. Biol. Chem. 271, 646-652 (1996).

12. Pape, T., Wintermeyer, W. \& Rodnina, M.V. Complete kinetic mechanism of elongation factor Tu-dependent binding of aminoacyl-tRNA to the A site of the E. coli ribosome. EMBO J. 17, 7490-7497 (1998).

13. Daviter, T., Wieden, H.-J. \& Rodnina, M.V. Essential role of histidine 84 in elongation factor Tu for the chemical step of GTP hydrolysis on the ribosome. J. Mol. Biol. 332 , 689-699 (2003).

14. Rodnina, M.V., Fricke, R., Kuhn, L. \& Wintermeyer, W. Codon-dependent conformational change of elongation factor Tu preceding GTP hydrolysis on the ribosome. EMBO J. 14, 2613-2619 (1995).

15. Thompson, R.C., Dix, D.B., Gerson, R.B. \& Karim, A.M. Effect of $\mathrm{Mg}^{2+}$ concentration, polyamines, streptomycin, and mutations in ribosomal proteins on the accuracy of the two-step selection of aminoacyl-tRNAs in protein biosynthesis. J. Biol. Chem. 
256, 6676-6681 (1981).

16. Lando, D., Cousin, M.A., Ojasoo, T. \& Raymond, J.P. Paromomycin and dihydrostreptomycin binding to Escherichia coli ribosomes. Eur. J. Biochem. 66, 597-606 (1976).

17. Bilgin, N. \& Ehrenberg, M. Mutations in $23 \mathrm{~S}$ ribosomal RNA perturb transfer RNA selection and can lead to streptomycin dependence. J. Mol. Biol. 235, 813-824 (1994).

18. Grise-Miron, L., Noreau, J., Melancon, P. \& Brakier-Gingras, L. Comparison of the misreading induced by streptomycin and neomycin. Biochim. Biophys. Acta 656 103-110 (1981).

19. Yates, J.L. Role of ribosomal protein S12 in discrimination of aminoacyl-tRNA J. Biol. Chem. 254, 11550-11554 (1979).

20. Campuzano, S., Cabanas, M.J. \& Modolell, J. The binding of non-cognate Tyr-tRNATyr to poly(uridylic acid)-programmed Escherichia coli ribosomes. Eur. J. Biochem. 100, 133-139 (1979).

21. Fersht, A. Structure and Mechanism in Protein Science 630 (Freeman, New York, 1998).

22. Ehrenberg, M., Bilgin, N. \& Kurland, C.G. Design and use of a fast and accurate in vitro translation system. In Ribosomes and Protein Biosynthesis. The Practical Approach Series (ed. Spedding, G.) 101-129 (IRL, Oxford, 1990).

23. Thompson, R.C. \& Stone, P.J. Proofreading of the codon-anticodon interaction on ribosomes. Proc. Natl. Acad. Sci. USA 74, 198-202 (1977).

24. Belanger, F., Leger, M., Saraiya, A.A., Cunningham, P.R. \& Brakier-Gingras, L. Functional studies of the 900 tetraloop capping helix 27 of $16 \mathrm{~S}$ ribosomal RNA. J. Mol. Biol. 320, 979-989 (2002).

25. O'Connor, M., Thomas, C.L., Zimmermann, R.A. \& Dahlberg, A.E. Decoding fidelity at the ribosomal $A$ and $P$ sites: influence of mutations in three different regions of the decoding domain in 16S rRNA. Nucleic Acids Res. 25, 1185-1193 (1997).

26. Gregory, S.T., Bayfield, M.A., O'Connor, M., Thompson, J. \& Dahlberg, A.E. Probing ribosome structure and function by mutagenesis. Cold Spring Harb. Symp. Quant Biol. 66, 101-108 (2001).

27. Gregory, S.T., Cate, J.H. \& Dahlberg, A.E. Streptomycin-resistant and streptomycindependent mutants of the extreme thermophile Thermus thermophilus. J. Mol. Biol. 309, 333-338 (2001).

28. Robert, F. \& Brakier-Gingras, L. A functional interaction between ribosomal proteins S7 and S11 within the bacterial ribosome. J. Biol. Chem. 278, 44913-44920 (2003).

29. Vila-Sanjurjo, A. et al. X-ray crystal structures of the WT and a hyper-accurate ribosome from Escherichia coli. Proc. Natl. Acad. Sci. USA 100, 8682-8687 (2003).

30. Stark, H. et al. Ribosome interactions of aminoacyl-tRNA and elongation factor Tu in the codon recognition complex. Nat. Struct. Biol. 9, 849-854 (2002).
31. Valle, M. et al. Cryo-EM reveals an active role for aminoacyl-tRNA in the accommodation process. EMBO J. 21, 3557-3567 (2002).

32. Valle, M. et al. Incorporation of aminoacyl-tRNA into the ribosome as seen by cryoelectron microscopy. Nat. Struct. Biol. 10, 899-906 (2003).

33. Vorstenbosch, E., Pape, T., Rodnina, M.V., Kraal, B. \& Wintermeyer, W. The G222D mutation in elongation factor Tu inhibits the codon- induced conformational changes leading to GTPase activation on the ribosome. EMBO J. 15, 6766-6774 (1996)

34. Yarus, M., Valle, M. \& Frank, J. A twisted tRNA intermediate sets the threshold for decoding. RNA 9, 384-385 (2003).

35. Yarus, M. \& Smith, D. tRNA on the ribosome: a waggle theory. In $t R N A$ : Structure, Biosynthesis, and Function (ed. RajBhandary, U.) 443-468 (American Society for Microbiology, Washington, DC, 1995).

36. Rodnina, M.V., Fricke, R. \& Wintermeyer, W. Transient conformational states of aminoacyl-tRNA during ribosome binding catalyzed by elongation factor Tu. Biochemistry 33, 12267-12275 (1994).

37. Piepenburg, $\mathrm{O}$. et al. Intact aminoacyl-tRNA is required to trigger GTP hydrolysis by elongation factor Tu on the ribosome. Biochemistry 39, 1734-1738 (2000).

38. Vogeley, L., Palm, G.J., Mesters, J.R. \& Hilgenfeld, R. Conformational change of elongation factor Tu (EF-Tu) induced by antibiotic binding. Crystal structure of the complex between EF-Tu-GDP and aurodox. J. Biol. Chem. 276, 17149-17155. (2001).

39. Mohr, D., Wintermeyer, W. \& Rodnina, M.V. GTPase activation of elongation factors Tu and $\mathrm{G}$ on the ribosome. Biochemistry 41, 12520-12528 (2002).

40. Vetter, I.R. \& Wittinghofer, A. The guanine nucleotide-binding switch in three dimensions. Science 294, 1299-1304 (2001).

41. Gromadski, K.B., Wieden, H.J. \& Rodnina, M.V. Kinetic mechanism of elongation factor Ts-catalyzed nucleotide exchange in elongation factor Tu. Biochemistry 41, $162-169$ (2002).

42. Calogero, R.A., Pon, C.L., Canonaco, M.A. \& Gualerzi, C.O. Selection of the mRNA translation initiation region by Escherichia coli ribosomes. Proc. Natl. Acad. Sci. USA 85, 6427-6431 (1988).

43. Rodnina, M.V. \& Wintermeyer, W. GTP consumption of elongation factor Tu during translation of heteropolymeric mRNAs. Proc. Natl. Acad. Sci. USA 92, 1945-1949 (1995).

44. Katunin, V.I., Muth, G.W., Strobel, S.A., Wintermeyer, W. \& Rodnina, M.V. Important contribution to catalysis of peptide bond formation by a single ionizing group within the ribosome. Mol. Cell 10, 339-346 (2002).

45. Stern, S., Moazed, D. \& Noller, H.F. Structural analysis of RNA using chemical and enzymatic probing monitored by primer extension. Methods Enzymol. 164, 481-489 (1988).

46. Moazed, D. \& Noller, H.F. Interaction of antibiotics with functional sites in $16 \mathrm{~S}$ ribosomal RNA. Nature 327, 389-394 (1987). 